\title{
CHARACTERISTICS OF DOUGH AND BREAD AS AFFECTED BY THE INCORPORATION OF SWEET POTATO PASTE IN THE FORMULATION
}

\author{
Kun-Lun Wu \\ Department of Hotel \& Restaurant Management, Chia Nan University of Pharmacy and Science, Tainan, R.O.C. \\ Wen-Chieh Sung \\ Department of Hotel \& Restaurant Management, Chia Nan University of Pharmacy and Science, Tainan, R.O.C., \\ sungwilliam2001@yahoo.com.tw \\ Chuan-Hua Yang \\ Department of Restaurant, Hotel and Institutional Management, Fu Jen Catholic University, Taipei County, R.O.C.
}

Follow this and additional works at: https://jmstt.ntou.edu.tw/journal

Part of the Life Sciences Commons

\section{Recommended Citation}

Wu, Kun-Lun; Sung, Wen-Chieh; and Yang, Chuan-Hua (2009) "CHARACTERISTICS OF DOUGH AND BREAD AS AFFECTED BY THE INCORPORATION OF SWEET POTATO PASTE IN THE FORMULATION," Journal of Marine Science and Technology. Vol. 17: Iss. 1, Article 3.

DOI: 10.51400/2709-6998.1972

Available at: https://jmstt.ntou.edu.tw/journal/vol17/iss1/3

This Research Article is brought to you for free and open access by Journal of Marine Science and Technology. It has been accepted for inclusion in Journal of Marine Science and Technology by an authorized editor of Journal of Marine Science and Technology. 


\title{
CHARACTERISTICS OF DOUGH AND BREAD AS AFFECTED BY THE INCORPORATION OF SWEET POTATO PASTE IN THE FORMULATION
}

\author{
Kun-Lun $\mathrm{Wu}^{*}$, Wen-Chieh Sung*, and Chuan-Hua Yang**
}

Key words: sweet potato, toast, texture profile analysis (TPA), sensory evaluation.

\begin{abstract}
Effects of four sweet potato (TNu57, TNu62, TNu64, and TNu66) pastes $(5 \%, 10 \%, 20 \%$, and $30 \%$ of wheat flour) on physicochemical properties of dough and toast were investigated. Farinograph, extensigraph, alveogarph, amylograph, dough expansion, baking test, and sensory of toast were evaluated. Farinograph absorption and mixing time all decreased as the addition of sweet potato paste increased $(\mathrm{p}<0.05)$. According to the results of alveograph, the tenacity, extensibility, and deformation energy will decrease with the increase of sweet potato paste $(p<0.05)$. Loaf volume slightly decreases with addition more than $20 \%$ of sweet potato paste. Ten percentage of sweet potato paste could be added in dough without decreasing dough expansion and volume of toast. The lowest hardness of texture was observed for 20\% TNu57 sweet potato paste addition. Results of this research suggest addition of TNu57 sweet potato paste, which contains high amount of maltose, was best variety for toast making. The addition of sweet potato paste exhibited higher staling rate than the control, but the hardness of bread is softer than the control at baking day $(\mathrm{p}<0.05)$ except TNu64. Toast supplemented with sweet potato paste scored more favorable than that of the control by panelists $(\mathrm{p}<0.05)$.
\end{abstract}

\section{INTRODUCTION}

Sweet potatoes (Ipomea batatas (L.) Lam.) play an important secondary food crop in developing countries [24]. Sweet potatoes and yams (Dioscorea species) are generally used interchangeably. However, they are from very different plant families. Their importance is not duly recognized and there is little

Paper submitted 05/15/07; accepted 01/08/08. Author for correspondence: Wen-Chieh Sung (e-mail:sungwilliam2001@yahoo.com.tw).

* Department of Hotel \& Restaurant Management, Chia Nan University of Pharmacy and Science, Tainan, R.O.C.

** Department of Restaurant, Hotel and Institutional Management, Fu Jen Catholic University, Taipei County, R.O.C. information and statistic on the extent of their cultivation, yield, production, marketing, storage, and utilization methods, although there has been a steady increase in the area planted with sweet potato from about 55,000 hectares in 1988 to about 65,000 hectares in 1996 [9]. The sweet potato is a nutritious and economical food, particularly the yellow and orange flesh varieties. They are low in fat, high in energy, fiber, vitamin C, potassium, $\beta$-carotene, and their protein yield are far higher than the cassava $[11,25,35]$. There are some data regarding the bread supplemented with sweet potato flour $[10,12,21]$ and yam flour $[4,13,20,23]$. Sweet potato flour composite bread generally has a relatively strong undesirable flavor and it greatly affects its quality and commercial [17]. Chen [3] reported twenty percentage of sweet potato flour could be added in dough without decreasing volume of bread. Loaf volume significantly decreases with the addition more than $20 \%$ of sweet potato flour.

In this study, physicochemical properties of 4 sweet potato paste composite bread were investigated in an attempt to relate these parameters to the performance of sweet potato paste in bread baking. Differences between the composite bread and the conventional bread have potential to improve the processed stability and quality of sweet potato bread.

\section{MATERIALS AND METHODS}

\section{Materials}

Bread flours were obtained from Cha Hwa Coporation (Taichung, Taiwan). Compressed yeast was purchased from Yung Cheng Industries Ltd. (Taipei, Taiwan). Two sweet potato varieties (Tainung No. 62 (TNu62), Tainung No. 64 (TNu64)) used in this study were purchased from Taoyuan City Farmers' Association (Taoyuan city, Taiwan). The other two varieties, Tainung No. 57 (TNu57) and Tainung No. 66 (TNu66), were purchased from Yih Thai grocery store (Taipei, Taiwan). Shortening used in this study was purchased from Namchow group (Taoyuan, Taiwan).

\section{Methods}

1) The Composition of Bread Flour and Sweet Potato Crude protein, ash, moisture content of bread flour were 
measured according AACC procedures 46-12, 08-01, and 44-15A, respectively [1]. The ash content, lipid content, simple sugars, and crude protein of sweet potato paste were determined by following AACC standard methods to be 08-01, 30-10, 80-04, and 46-12, respectively [1]. The moisture content of sweet potato was conducted following AOAC procedure 984.25 [2]. Proximate composition and simple sugars of sweet potato were performed on each variety by using three replicates. One replicate of sweet potato was subdivided into 3 samples. Sweet potato paste production included washing, hand peeling, and shredding the raw sweet potato roots into a $0.5 \mathrm{~cm}$ thick. The shredded sweet potatoes were steamed for half an hour and they were blended with a mixer (Hobart, TryOhio, USA) attached with a paddle for 5 minutes. The sweet potato paste was cooled and stored in airtight containers at $-18^{\circ} \mathrm{C}$ until used.

\section{2) Physicochemical Properties of Dough}

Bread flour was blended with the thawed sweet potato paste individually in ratios of $0 \mathrm{~g} / 100 \mathrm{~g}$ (sweet potato paste/bread flour), 5g/95g (5\%), 10g/90g (10\%), 20g/80g (20\%), 30g/70g (30\%). Farinographs (C.W. Brabender instruments, Inc., South Hackensach, NJ) were run at $30^{\circ} \mathrm{C}$ and the bread dough was made from $300 \mathrm{~g}$ bread flour (or sweet potato paste/bread flour) and added water to center at 500 Brabender units by adjusting dough water content. It is used to access flour water absorption, mixing time, and mixing tolerance index (MTI) according to AACC method 54-21 [1]. The Brabender Extensigraph (South Hackensack, NJ) was used to measure dough extensibility $(\mathrm{cm})$ and resistance to stretching (BU) after 45 minutes and 90 minutes rest period. Extensigraph of dough was measured following the method of AACC 54-10 [1]. Brabender Fariongraph and Extensigraph tests were performed on each sweet potato level by using three replicates. All the mean values and standard deviations were reported from three replicates, one replicate is the mean of three determinations of the level.

Amylograph viscosity of various groups was measured in triplicate using a Brabender Instrument (C.W. Brabender Instruments Inc., South Hackensack, New Jersey) Visco/Amylo/ Graph. A 9g/100g slurry of 40.5 gram flour (or sweet potato paste/bread flour) and $450 \mathrm{ml}$ distilled water and various percentage of sweet potato paste based on flour weight was heated from $30^{\circ} \mathrm{C}$ to $95^{\circ} \mathrm{C}$ at a rate of $1.5^{\circ} \mathrm{C}$ per minute, held at $95^{\circ} \mathrm{C}$ for 60 minutes and then cooled at a rate of $1.5^{\circ} \mathrm{C}$ to $50^{\circ} \mathrm{C}$. Peak viscosity (BU) and temperature at peak were recorded following the method of AACC 22-10 [1]. Alveograph characteristics of bread flour and test groups using Chopin Alveograph (Model Alveographe NG, Chopin, France) were determined using AACC methods [1]. Dough was mixed in the alveograph mixer (Chopin, France) and then extruded as a sheet. Disks are cut from the sheet and, after a rest period $\left(28\right.$ minutes, $\left.25.5^{\circ} \mathrm{C}\right)$, they are clamped down and inflated with air. The work of the deformation energy (W value), length of the curve (L), maximum height $(\mathrm{P})$, and $\mathrm{P} / \mathrm{L}$ value were recorded..

\section{3) Dough Expansion}

Dough expansion test followed the method of Sangnark and Noomhorm [26] with slight modification. Dough after mixing was divided into 50 gram pieces and rounded. Dough samples were inserted into a $500 \mathrm{ml}$ graduated cylinder. Cylinders were placed in a cabinet at a temperature of $29^{\circ} \mathrm{C}$ and relative humidity (RH) of $75-80 \%$ for 210 minutes. Dough volume was recorded every 30 minutes. A straight dough procedure (AACC method 10-09) was used for baking method [1]. The baking formula, based on baker's percentage, was as follows, $100 \%$ bread flour, $6 \%$ sugar, $1.5 \%$ salt, $5.3 \%$ compressed yeast, $3 \%$ shortening, $0-30 \%$ sweet potato paste, and water as determined from a farinograph absorption test.

Appearance and uniformity of bread were evaluated by the method of Kruger et al. [15]. A color meter (Hunter Lab Color Flex CX 0407, Model No. 45/0, Hunter Associates, Inc., VA. USA) was used to determine lightness (L), red content (a) and yellow content (b) values on bread crumb at first day. A white and a black reference plates were used as a standard. White index (WI) was calculated as following formula:

$$
\mathrm{WI}=100-\left((100-\mathrm{L})^{2}+\mathrm{a}^{2}+\mathrm{b}^{2}\right)^{1 / 2} .
$$

Loaf volume was measured after baking by a rapeseed displacement method. Specific volume was calculated as loaf volume $\left(\mathrm{cm}^{3}\right) /$ loaf weight (gram) [27].

4) Texture Profile Analysis (TPA)

Toasts were sliced into $2.5 * 2.5 * 2.5 \mathrm{~cm}^{3}$ crumbs using a standard bread slicer. Hardness of toasts were tested with the TA.XT2 Texture Analyzer (Stable Micro Systems Co., Ltd., Haslemere, England) and a No P/0.5S/0.5" Diameter cylinder probe according to the methods of Wang and Sun [32]. Test speed was $10 \mathrm{~mm} /$ minute, trigger force was $20 \mathrm{~g}$, distance was $10 \mathrm{~mm}$ (40\% compression) and wait time between first and second compression cycle was 5 seconds. Toasts were sealed in $1 \mathrm{~kg}$ polyethylene (PE) bags after cooling and held at room temperature $\left(25^{\circ} \mathrm{C}\right)$ for further testing. Toasts were sliced into crumbs and hardness was recorded for 3 days storage duration. 5) Sensory Evaluation

Tested groups and control toasts were served to 20 panelists to evaluate texture, color and overall acceptability scores. Twenty male and female students of department of Hotel \& Restaurant Management between the ages of 18 and 22 were participants on the panel. Panelists were instructed to evaluate each attribute using a seven point hedonic scale. Toasts coded with a three digits were supplied to them. Each data point from sensory analysis represents an average of twenty panelists.

6) Statistical Analysis

All determinations were replicated three times, mean values and standard deviations were reported. Analysis of variance (ANOVA) was performed by Ducan's test $(\mathrm{p}<0.05)$ using the Statistical Analysis System [28]. When the difference in ANOVA among the hardness of samples was significant, pair comparison of these samples were analyzed using Tukey's test.

\section{RESULTS AND DISCUSSION}

Moisture, crude protein, ash, wet gluten, and dried gluten content of bread flour was $13.29 \%, 13.13 \%, 0.50 \%, 38.90 \%$, and $13.50 \%$, respectively. The results of the proximate composition of sweet potato paste are presented in Table 1 . The 
Table 1. Proximate composition* of four potato varieties.

\begin{tabular}{llcccccccc}
\hline Varieties & \multirow{2}{*}{ Color } & Moisture & Ash & Crude protein Crude fat & Crude fiber & Starch & Sucrose & Maltose \\
\cline { 2 - 8 } & & \multicolumn{7}{c}{$(\%)$} \\
\hline TNu57 & Orange yellow & $67.10 \pm 0.94^{\mathrm{bc}}$ & $0.95 \pm 0.01^{\mathrm{c}}$ & $0.38 \pm 0.01^{\mathrm{c}}$ & $2.03 \pm 0.04^{\mathrm{b}}$ & $1.25 \pm 0.03^{\mathrm{a}}$ & $25.85 \pm 0.98^{\mathrm{a}}$ & $3.30 \pm 0.12^{\mathrm{b}}$ & $10.41 \pm 0.22^{\mathrm{a}}$ \\
TNu62 & Orange red & $65.02 \pm 0.52^{\mathrm{c}}$ & $1.12 \pm 0.03^{\mathrm{b}}$ & $0.39 \pm 0.01^{\mathrm{c}}$ & $2.05 \pm 0.07^{\mathrm{b}}$ & $1.21 \pm 0.02^{\mathrm{a}}$ & $28.43 \pm 0.85^{\mathrm{a}}$ & $3.10 \pm 0.09^{\mathrm{bc}}$ & $4.89 \pm 0.07^{\mathrm{b}}$ \\
TNu64 & Dark orange & $70.12 \pm 0.81^{\mathrm{b}}$ & $1.23 \pm 0.02^{\mathrm{a}}$ & $0.51 \pm 0.02^{\mathrm{a}}$ & $2.51 \pm 0.03^{\mathrm{a}}$ & $1.02 \pm 0.03^{\mathrm{b}}$ & $22.61 \pm 0.46^{\mathrm{b}}$ & $2.91 \pm 0.04^{\mathrm{c}}$ & $3.14 \pm 0.12^{\mathrm{c}}$ \\
TNu66 & Orange red & $75.30 \pm 0.56^{\mathrm{a}}$ & $1.01 \pm 0.02^{\mathrm{c}}$ & $0.46 \pm 0.01^{\mathrm{b}}$ & $2.10 \pm 0.03^{\mathrm{b}}$ & $0.97 \pm 0.01^{\mathrm{b}}$ & $18.10 \pm 0.23^{\mathrm{c}}$ & $4.51 \pm 0.08^{\mathrm{a}}$ & $4.72 \pm 0.18^{\mathrm{b}}$
\end{tabular}

*Mean \pm Standard deviation, $n=3$. Means with the same column are not significantly different at $5 \%$ level.

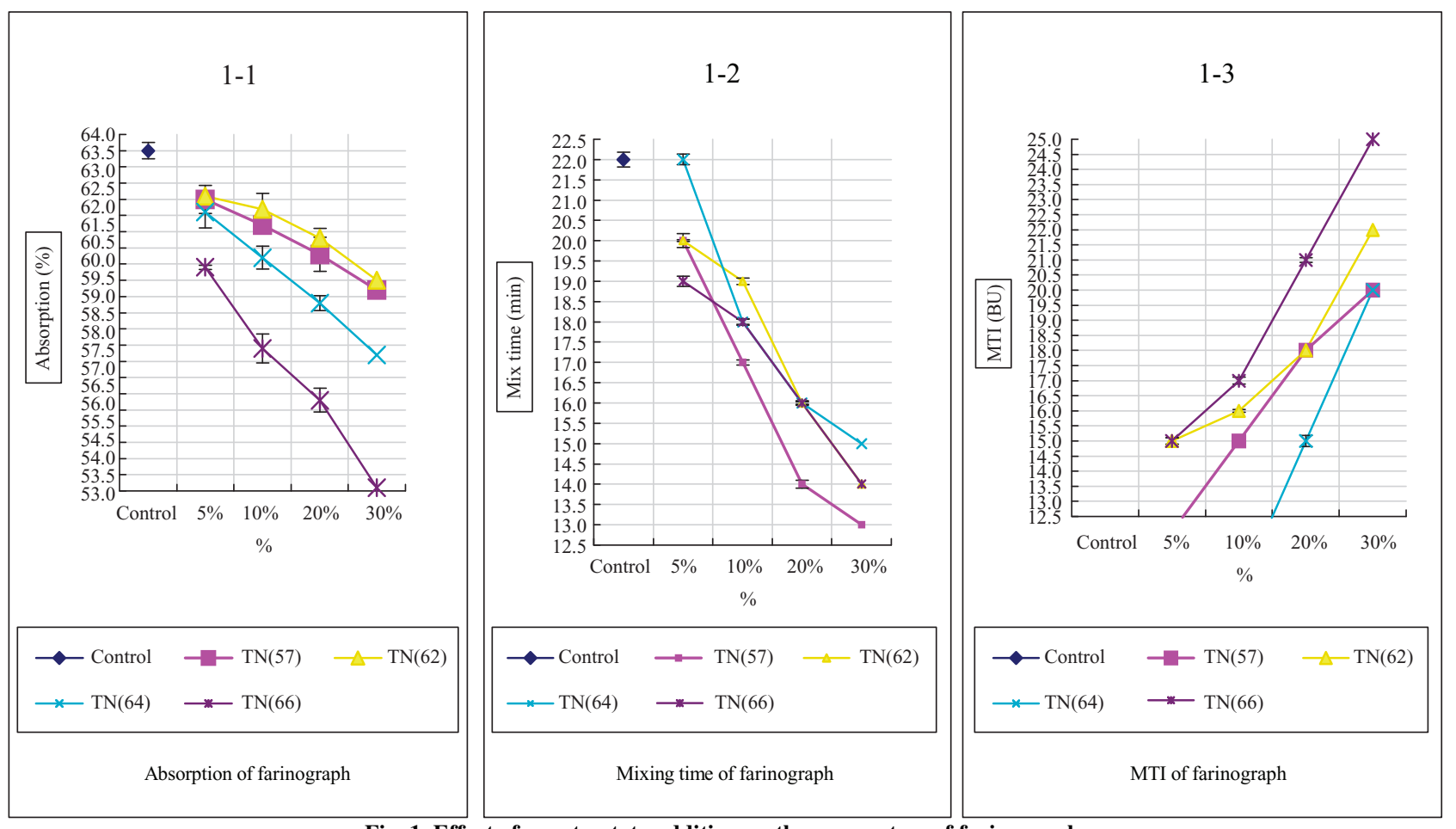

Fig. 1. Effect of sweet potato addition on the parameters of farinograph.

TNu66 sweet potato showed highest moisture content $(75.30 \%)$ and lowest starch content $(18.10 \%)$ than the other varieties. To determine sugar contents in sweet potato paste, HPLC analyses were performed; sucrose and maltose contents were determined. HPLC analysis shows that TNu57 sweet potato contains high amount of maltose (10.41\%) (Table 1).

Farinograph curves (farinograms) from the additions of various sweet potato paste are shown in Fig. 1. Parameters (absorption, mixing time and mixing tolerance indes (MTI)) of farinograms with different ratio of sweet potato paste were also summarized at Table 2. Generally, addition of sweet potato paste resulted in a decrease of water absorption $(\mathrm{p}<0.05)$ in a farinograph curve, which is due to the high moisture content of sweet potato paste diluting the bread flour. Farinograph absorption is influenced primarily by flour protein content and starch damage $[6,31]$. The Farinograph absorptions of addition TNu66 sweet potato groups are lowest among all sweet potato varieties. This decrease in absorption may be due to TNu66 sweet potato paste containing more moisture (Table 1). It shows the addition of sweet potato paste decreases the expansion and extensibility of the dough. All dough strengths of addition sweet potato paste were weaker than the control dough (Table 2).

The alveograph test is highly sensitive to flour starch damage, and the result may be drastically influenced by flour starch damage [6]. The alveograph may give misleading results for hard wheat, but it still provides an estimate of physical dough properties. All above parameters will affect the process, molding and even the appearance and internal structure of bread. The addition of sweet potato paste showed the dough will relax well for higher amount of sweet potato paste addition for the same intermediate proof period. Results of alveograph showed that the greatest variety of dough supplemented with sweet potato is TNu57. An increase of water absorption has been reported by Lazaridou et al. [16] with various hydrocolloids, such as pectin, carboxymethylcellulose, agarose, and xanthan, were added to wheat flour. The most distinctive change on addition of sweet potato paste is the increase in Mixing Tolerance Index of farinograph, the drop in curve consistency five minutes past the peak and by measuring the stability. Mixing 
Table 2. Effect of sweet potato paste addition on the rheology property of dough.

\begin{tabular}{|c|c|c|c|c|c|c|c|c|c|c|c|c|c|}
\hline \multirow{3}{*}{ Sample } & \multicolumn{3}{|c|}{ Farinograph } & \multicolumn{4}{|c|}{ Alveography } & \multicolumn{4}{|c|}{ Extensigraph } & \multicolumn{2}{|c|}{ Amylograph } \\
\hline & \multirow{2}{*}{$\frac{\text { Absorption }}{(\%)}$} & \multirow{2}{*}{$\begin{array}{c}\begin{array}{c}\text { Mixing } \\
\text { time }\end{array} \\
(\min )\end{array}$} & \multirow{2}{*}{$\begin{array}{l}\text { MTI } \\
(\mathrm{BU})\end{array}$} & $\mathrm{P}$ & $\mathrm{L}$ & $\mathrm{P} / \mathrm{L}$ & \multirow[t]{2}{*}{ W } & \multicolumn{2}{|c|}{$45 \mathrm{~min}$} & \multicolumn{2}{|c|}{$90 \mathrm{~min}$} & \multirow{2}{*}{$\begin{array}{c}\text { Peak } \\
(\mathrm{BU})\end{array}$} & \multirow{2}{*}{$\begin{array}{c}\begin{array}{c}\text { Temp. at } \\
\text { peak }\end{array} \\
\left({ }^{\circ} \mathrm{C}\right)\end{array}$} \\
\hline & & & & $\mathrm{mmH}_{2} \mathrm{O}$ & $\mathrm{mm}$ & $\mathrm{mmH}_{2} \mathrm{O} / \mathrm{mm}$ & & $\begin{array}{l}\text { Exten.* } \\
(\mathrm{cm})\end{array}$ & $\begin{array}{c}\text { Resis.** } \\
\text { (BU) }\end{array}$ & $\begin{array}{c}\text { Exten.* } \\
(\mathrm{cm})\end{array}$ & $\begin{array}{c}\text { Resis.** } \\
\text { (BU) }\end{array}$ & & \\
\hline Control & $63.5 \pm 0.25^{\mathrm{a}}$ & $22 \pm 0.18^{\mathrm{a}}$ & $2 \pm 0.02^{\mathrm{g}}$ & $89 \pm 0.53^{\mathrm{a}}$ & $131 \pm 0.13^{\mathrm{b}}$ & $0.69 \pm 0.01^{\mathrm{b}}$ & $394 \pm 0.39 a$ & $18.0 \pm 0.02^{\mathrm{c}}$ & $670 \pm 3.35^{\mathrm{g}}$ & $13.7 \pm 0.07^{\mathrm{b}}$ & $1000 \pm 9.00$ & $460 \pm 0.92^{\mathrm{d}}$ & $86.5 \pm 0.43^{\mathrm{a}}$ \\
\hline \multirow[t]{4}{*}{ TNu57 } & $62.0 \pm 0.31^{\mathrm{b}}$ & $20 \pm 0.18^{\mathrm{b}}$ & $12 \pm 0.11^{\mathrm{e}}$ & $79 \pm 0.47^{b}$ & $119 \pm 0.36^{\mathrm{c}}$ & $0.67 \pm 0.00^{\mathrm{c}}$ & $334 \pm 0.33^{\mathrm{b}}$ & $18.0 \pm 0.09^{\mathrm{c}}$ & $700 \pm 3.50^{f}$ & $13.5 \pm 0.07^{\mathrm{b}}$ & $>1000$ & $440 \pm 0.88^{\mathrm{e}}$ & $86.5 \pm 0.35^{\mathrm{a}}$ \\
\hline & $61.2 \pm 0.43^{\mathrm{b}}$ & $17 \pm 0.02^{\mathrm{d}}$ & $15 \pm 0.02^{\mathrm{d}}$ & $74 \pm 0.59^{c}$ & $122 \pm 0.73^{\mathrm{c}}$ & $0.63 \pm 0.00^{\mathrm{d}}$ & $332 \pm 1.00^{\mathrm{b}}$ & $15.7 \pm 0.14^{\mathrm{f}}$ & $810 \pm 5.67^{\mathrm{c}}$ & $12.5 \pm 0.09^{\mathrm{c}}$ & $>1000$ & $480 \pm 1.44^{\mathrm{b}}$ & $85.2 \pm 0.51^{\mathrm{a}}$ \\
\hline & $60.3 \pm 0.24^{\mathrm{c}}$ & $14 \pm 0.06^{\mathrm{g}}$ & $18 \pm 0.07^{\mathrm{c}}$ & $71 \pm 0.07^{\mathrm{d}}$ & $114 \pm 0.57^{\mathrm{d}}$ & $0.64 \pm 0.01^{\mathrm{d}}$ & $315 \pm 1.89^{\mathrm{c}}$ & $17.7 \pm 0.07^{\mathrm{d}}$ & $735 \pm 2.94^{\mathrm{e}}$ & $14.2 \pm 0.06^{\mathrm{a}}$ & $940 \pm 7.52$ & $455 \pm 2.73^{\mathrm{de}}$ & $86.0 \pm 0.77^{\mathrm{a}}$ \\
\hline & $59.2 \pm 0.53^{\mathrm{c}}$ & $13 \pm 0.10^{\mathrm{i}}$ & $20 \pm 0.18^{\mathrm{b}}$ & $70 \pm 0.42^{\mathrm{d}}$ & $85 \pm 0.19^{\mathrm{g}}$ & $0.83 \pm 0.00^{\mathrm{a}}$ & $255 \pm 0.26^{\mathrm{e}}$ & $16.8 \pm 0.15^{\mathrm{e}}$ & $635 \pm 5.72^{\mathrm{h}}$ & $12.9 \pm 0.12^{\mathrm{c}}$ & $730 \pm 1.46$ & $470 \pm 2.35^{\mathrm{c}}$ & $86.0 \pm 0.26^{\mathrm{a}}$ \\
\hline \multirow[t]{4}{*}{ TNu62 } & $62.1 \pm 0.31^{\mathrm{b}}$ & $20 \pm 0.06^{\mathrm{b}}$ & $15 \pm 0.06^{\mathrm{d}}$ & $74 \pm 0.07^{c}$ & $147 \pm 1.03^{\mathrm{a}}$ & $0.51 \pm 0.00^{\mathrm{f}}$ & $337 \pm 2.02^{\mathrm{b}}$ & $18.0 \pm 0.11^{\mathrm{c}}$ & $600 \pm 3.00^{\mathrm{i}}$ & $12.5 \pm 0.06^{\mathrm{c}}$ & $980 \pm 7.84$ & $463 \pm 0.46^{\mathrm{d}}$ & $86.0 \pm 0.69^{\mathrm{a}}$ \\
\hline & $61.7 \pm 0.06^{\mathrm{b}}$ & $19 \pm 0.17^{\mathrm{e}}$ & $16 \pm 0.10^{\mathrm{d}}$ & $68 \pm 0.41^{\mathrm{d}}$ & $114 \pm 0.46^{\mathrm{d}}$ & $0.60 \pm 0.00^{\mathrm{d}}$ & $276 \pm 0.28^{\mathrm{d}}$ & $19.3 \pm 0.04^{\mathrm{b}}$ & $675 \pm 0.68^{\mathrm{g}}$ & $12.6 \pm 0.01^{\mathrm{c}}$ & $1000 \pm 4.00$ & $445 \pm 3.12^{\mathrm{de}}$ & $86.5 \pm 0.26^{\mathrm{a}}$ \\
\hline & $60.8 \pm 0.49^{\mathrm{b}}$ & $16 \pm 0.08^{\mathrm{e}}$ & $18 \pm 0.05^{\mathrm{c}}$ & $59 \pm 0.18^{\mathrm{f}}$ & $102 \pm 0.10^{\mathrm{e}}$ & $0.61 \pm 0.00^{\mathrm{d}}$ & $212 \pm 1.70^{\mathrm{g}}$ & $19.3 \pm 0.17^{\mathrm{b}}$ & $625 \pm 5.00^{\mathrm{h}}$ & $14.9 \pm 0.12^{\mathrm{a}}$ & $780 \pm 0.78$ & $470 \pm 1.41^{\mathrm{c}}$ & $86.0 \pm 0.77^{\mathrm{a}}$ \\
\hline & $59.5 \pm 0.30^{\mathrm{c}}$ & $14 \pm 0.03^{\mathrm{h}}$ & $22 \pm 0.15^{\mathrm{b}}$ & $51 \pm 0.36^{\mathrm{g}}$ & $93 \pm 0.74^{\mathrm{f}}$ & $0.56 \pm 0.00^{\mathrm{e}}$ & $177 \pm 0.71^{\mathrm{i}}$ & $20.2 \pm 0.14^{\mathrm{a}}$ & $610 \pm 3.05^{\mathrm{i}}$ & $14.9 \pm 0.07^{\mathrm{a}}$ & $795 \pm 6.36$ & $490 \pm 3.92^{\mathrm{a}}$ & $86.5 \pm 0.52^{\mathrm{a}}$ \\
\hline \multirow[t]{4}{*}{ TNu64 } & $61.6 \pm 0.12^{\mathrm{b}}$ & $22 \pm 0.20^{\mathrm{a}}$ & $8 \pm 0.04^{\mathrm{f}}$ & $74 \pm 0.30^{c}$ & $127 \pm 0.76^{\mathrm{c}}$ & $0.59 \pm 0.00^{\mathrm{d}}$ & $309 \pm 0.31^{\mathrm{c}}$ & $17.8 \pm 0.07^{\mathrm{d}}$ & $855 \pm 1.71^{b}$ & $14.6 \pm 0.03^{\mathrm{a}}$ & $>1000$ & $460 \pm 2.30^{\mathrm{d}}$ & $86.0 \pm 0.17^{\mathrm{a}}$ \\
\hline & $60.2 \pm 0.48^{\mathrm{c}}$ & $18 \pm 0.13^{\mathrm{d}}$ & $10 \pm 0.03^{\mathrm{e}}$ & $65 \pm 0.13^{\mathrm{e}}$ & $124 \pm 0.50^{\mathrm{c}}$ & $0.53 \pm 0.00^{f}$ & $278 \pm 2.50^{\mathrm{d}}$ & $17.8 \pm 0.04^{\mathrm{d}}$ & $795 \pm 5.57^{\mathrm{c}}$ & $13.1 \pm 0.09^{b}$ & $>1000$ & $460 \pm 1.38^{\mathrm{d}}$ & $86.2 \pm 0.60^{\mathrm{a}}$ \\
\hline & $58.8 \pm 0.35^{\mathrm{c}}$ & $16 \pm 0.06^{\mathrm{e}}$ & $15 \pm 0.02^{\mathrm{d}}$ & $57 \pm 0.46^{\mathrm{f}}$ & $113 \pm 0.23^{\mathrm{d}}$ & $0.52 \pm 0.00^{\mathrm{f}}$ & $237 \pm 1.42^{\mathrm{f}}$ & $18.5 \pm 0.15^{\mathrm{c}}$ & $765 \pm 4.59^{d}$ & $13.8 \pm 0.07^{\mathrm{b}}$ & $>1000$ & $475 \pm 0.48^{\mathrm{c}}$ & $86.0 \pm 0.43^{\mathrm{a}}$ \\
\hline & $57.2 \pm 0.23^{\mathrm{d}}$ & $15 \pm 0.03^{\mathrm{f}}$ & $20 \pm 0.18^{\mathrm{b}}$ & $43 \pm 0.26^{\mathrm{h}}$ & $113 \pm 1.02^{\mathrm{d}}$ & $0.40 \pm 0.00^{\mathrm{h}}$ & $180 \pm 0.72^{\mathrm{i}}$ & $18.7 \pm 0.13^{\mathrm{c}}$ & $670 \pm 2.68^{\mathrm{g}}$ & $14.5 \pm 0.06^{\mathrm{a}}$ & $880 \pm 5.28$ & $470 \pm 4.23^{\mathrm{c}}$ & $86.0 \pm 0.26^{\mathrm{a}}$ \\
\hline \multirow[t]{4}{*}{ TNu66 } & $59.9 \pm 0.18^{\mathrm{c}}$ & $19 \pm 0.17^{\mathrm{c}}$ & $15 \pm 0.12^{\mathrm{d}}$ & $73 \pm 0.29^{c}$ & $123 \pm 0.86^{\mathrm{c}}$ & $0.60 \pm 0.01^{\mathrm{d}}$ & $313 \pm 0.63^{c}$ & $17.2 \pm 0.09^{\mathrm{d}}$ & $940 \pm 1.88^{\mathrm{a}}$ & $14.6 \pm 0.03^{\mathrm{a}}$ & $>1000$ & $455 \pm 3.19^{\mathrm{de}}$ & $86.0 \pm 0.09^{\mathrm{a}}$ \\
\hline & $57.4 \pm 0.06^{\mathrm{d}}$ & $18 \pm 0.13^{\mathrm{d}}$ & $17 \pm 0.10^{\mathrm{c}}$ & $61 \pm 0.18^{f}$ & $119 \pm 0.71^{\mathrm{c}}$ & $0.51 \pm 0.00^{\mathrm{f}}$ & $270 \pm 2.43^{\mathrm{d}}$ & $19.0 \pm 0.08^{\mathrm{b}}$ & $730 \pm 0.73^{\mathrm{e}}$ & $11.7 \pm 0.11^{\mathrm{d}}$ & $1000 \pm 3.00$ & $472 \pm 2.83^{\mathrm{c}}$ & $86.5 \pm 0.78^{a}$ \\
\hline & $55.8 \pm 0.45^{\mathrm{e}}$ & $16 \pm 0.08^{\mathrm{e}}$ & $21 \pm 0.11^{\mathrm{b}}$ & $45 \pm 0.05^{\mathrm{h}}$ & $101 \pm 0.51^{\mathrm{e}}$ & $0.46 \pm 0.00^{\mathrm{g}}$ & $192 \pm 1.34^{\mathrm{h}}$ & $16.3 \pm 0.03^{\mathrm{e}}$ & $780 \pm 7.02^{c}$ & $13.5 \pm 0.11^{\mathrm{b}}$ & $985 \pm 1.97$ & $460 \pm 1.84^{\mathrm{d}}$ & $87.0 \pm 0.61^{\mathrm{a}}$ \\
\hline & $53.1 \pm 0.37^{\mathrm{f}}$ & $14 \pm 0.06^{\mathrm{g}}$ & $25 \pm 0.08^{\mathrm{a}}$ & $34 \pm 0.31^{\mathrm{i}}$ & $88 \pm 0.26^{\mathrm{g}}$ & $0.40 \pm 0.00^{\mathrm{h}}$ & $129 \pm 0.77^{\mathrm{j}}$ & $17.2 \pm 0.02^{\mathrm{d}}$ & $740 \pm 5.92^{\mathrm{e}}$ & $13.7 \pm 0.08^{\mathrm{b}}$ & $965 \pm 8.69$ & $488 \pm 1.46^{\mathrm{a}}$ & $86.0 \pm 0.52^{\mathrm{a}}$ \\
\hline
\end{tabular}

MTI=Mixing index, measured in Brabender Units (BU).

$\mathrm{P}=$ tenacity; $\mathrm{L}=$ extensibility; $\mathrm{P} / \mathrm{L}=$ distortion; $\mathrm{W}=$ deformation energy.

*Extensibility measured in centimeters $(\mathrm{cm})$.

***Resistance to extension measured in BU (Max).
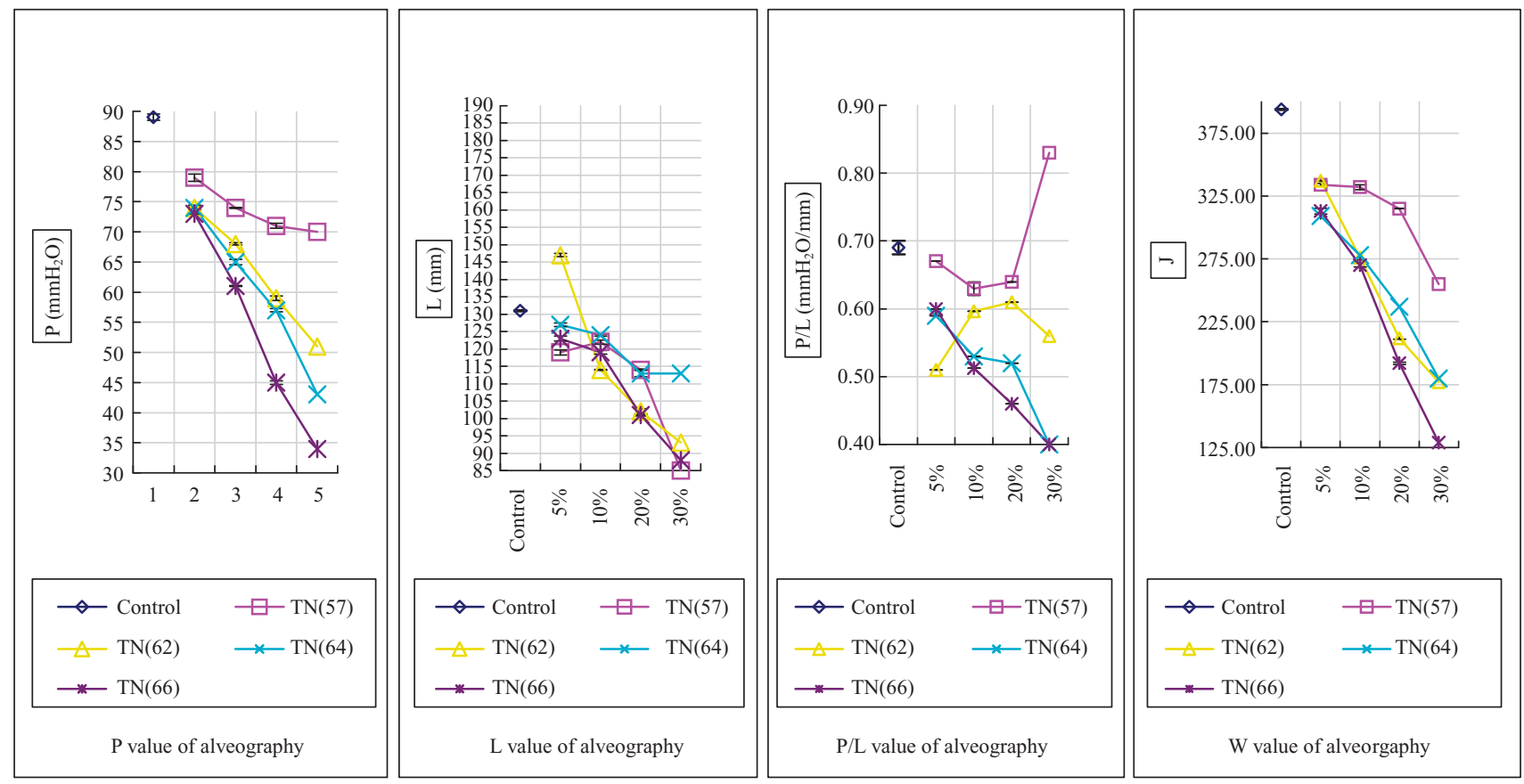

Fig. 2. Effect of sweet potato paste of the parameters of alveograph.

tolerance was defined as the interval in minutes between the time the curve first reaches the 500 Brabender Unit (BU) line and the time the curve first drops below the $500 \mathrm{BU}$ line. Three sweet potato varieties, TNu57, TNu62, and TNu64 were considered as great ingredients for toast making processing. Alveograph curves (alveograms) for control and different ratio of sweet potato paste addition are shown in Fig. 2.
The $\mathrm{P}$ value (dough resistance to deformation or tenacity) is an indicator of the dough's ability to retain gas [33]. The $\mathrm{P}$ values decreased with the addition of the four different sweet potato pastes. The highest effect was exhibited by $30 \% \mathrm{TNu} 64$, and the lowest influence was by TNu57. This might be due to interactions between the fiber structure and the wheat proteins [14]. When sweet potato paste was added, the maximum height 

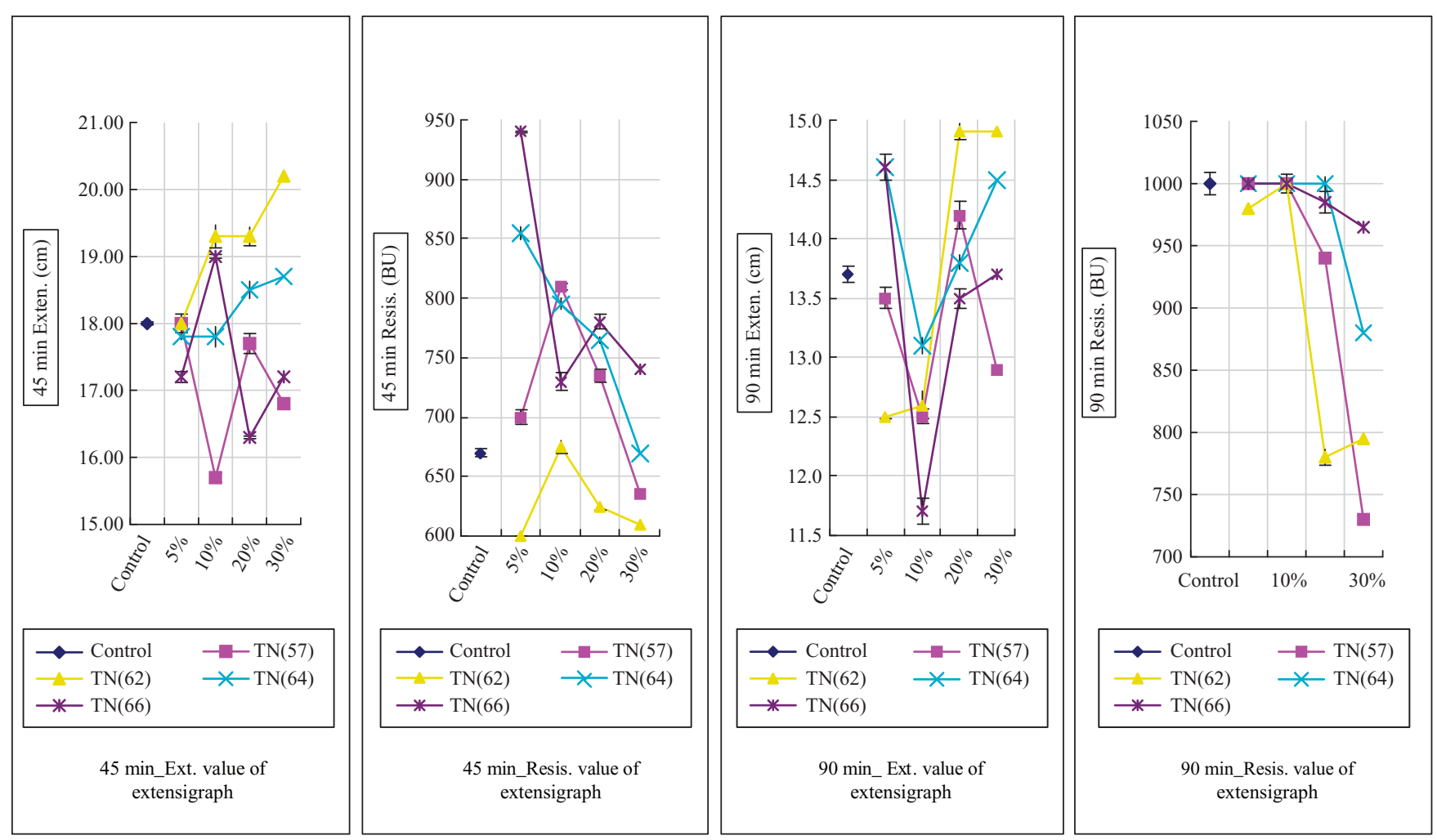

Fig. 3. Effect of sweet potato paste addition on the parameters of extensigram.
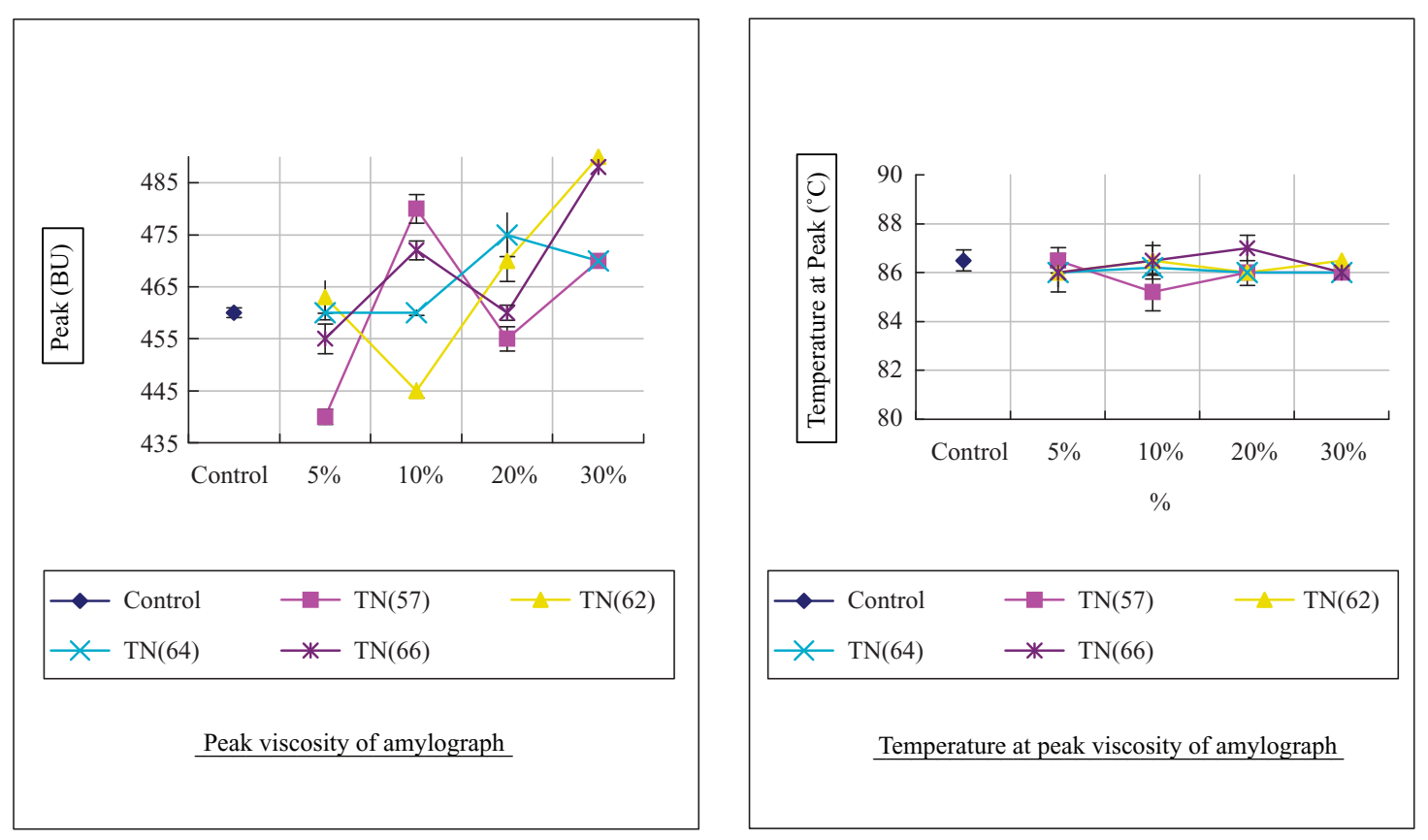

Fig. 4. Effect of sweet potato paste addition on peak viscosity and peak temperature of amylograph.

(tenacity; P value), the length of the curve (extensibility; L value), $\mathrm{P} / \mathrm{L}$ value (distortion) and $\mathrm{W}$ value (deformation energy) of alveogram decreased notably (Table 2). The length of the curve ( $\mathrm{L}$ value), a predictor of the processing characteristics of the dough, was also greatly reduced by adding sweet potato paste, with the exception of 5\% TNu62, which extended L value. The maximum height and the length of the alveograph curve are related to the resistance to expansion and to the extensibility of the dough. The $\mathrm{P} / \mathrm{L}$ ratio value provides information about the elastic resistance and extensibility balance of flour dough. The addition of TNu57 sweet potato paste led to the highest $\mathrm{P} / \mathrm{L}$ ratio range, probably due to the high content of fiber and maltose, which favors a strong interaction between fiber and the flour proteins. The work of deformation until rupture, or the "W" 
Table 3. Expansion of dough a various levels of sweet potato paste addition.

\begin{tabular}{|c|c|c|c|c|c|c|c|c|c|}
\hline \multirow[t]{2}{*}{ Varieties } & Addition $\%$ & $0 \mathrm{~min}$ & $30 \mathrm{~min}$ & $60 \mathrm{~min}$ & $90 \mathrm{~min}$ & $120 \mathrm{~min}$ & $150 \mathrm{~min}$ & $180 \mathrm{~min}$ & $210 \mathrm{~min}$ \\
\hline & \multicolumn{9}{|c|}{ Volume* $\left(\mathrm{cm}^{3}\right)$} \\
\hline \multirow[t]{5}{*}{ TNu57 } & 0 & $47 \pm 0.67^{\mathrm{ab}}$ & $60 \pm 1.92^{\mathrm{a}}$ & $92 \pm 0.83^{\mathrm{a}}$ & $128 \pm 5.38^{\mathrm{a}}$ & $168 \pm 5.21^{\mathrm{a}}$ & $190 \pm 7.03^{\mathrm{ab}}$ & $218 \pm 4.14^{\mathrm{a}}$ & $220 \pm 1.98^{\mathrm{a}}$ \\
\hline & 5 & $48 \pm 0.96^{\mathrm{a}}$ & $62 \pm 1.74^{\mathrm{a}}$ & $95 \pm 2.09^{\mathrm{a}}$ & $133 \pm 1.46^{\mathrm{a}}$ & $173 \pm 3.11^{\mathrm{a}}$ & $208 \pm 2.29^{\mathrm{a}}$ & $210 \pm 0.42^{\mathrm{b}}$ & $220 \pm 2.86^{\mathrm{a}}$ \\
\hline & 10 & $44 \pm 1.72^{\mathrm{c}}$ & $56 \pm 1.18^{b}$ & $82 \pm 2.54^{\mathrm{b}}$ & $113 \pm 4.52^{\mathrm{b}}$ & $145 \pm 2.03^{\mathrm{c}}$ & $173 \pm 5.02^{\mathrm{c}}$ & $170 \pm 3.91^{\mathrm{d}}$ & $172 \pm 5.50^{\mathrm{c}}$ \\
\hline & 20 & $46 \pm 1.89^{\mathrm{abc}}$ & $56 \pm .067^{\mathrm{b}}$ & $84 \pm 3.19^{\mathrm{b}}$ & $120 \pm 0.60^{\mathrm{b}}$ & $150 \pm 3.60^{\mathrm{bc}}$ & $168 \pm 7.22^{\mathrm{c}}$ & $163 \pm 6.52^{\mathrm{d}}$ & $168 \pm 2.35^{\mathrm{c}}$ \\
\hline & 30 & $45 \pm 0.18^{\mathrm{bc}}$ & $54 \pm 0.11^{\mathrm{b}}$ & $81 \pm 3.48^{\mathrm{b}}$ & $120 \pm 2.5^{\mathrm{b}}$ & $158 \pm 2.69^{\mathrm{b}}$ & $192 \pm 7.10^{\mathrm{b}}$ & $192 \pm 8.83^{c}$ & $182 \pm 6.34^{\mathrm{b}}$ \\
\hline \multirow[t]{5}{*}{ TNu62 } & 0 & $47 \pm 0.67^{\mathrm{a}}$ & $60 \pm 1.92^{\mathrm{a}}$ & $92 \pm 0.83^{\mathrm{a}}$ & $128 \pm 5.38^{\mathrm{a}}$ & $168 \pm 5.21^{\mathrm{a}}$ & $190 \pm 7.03^{b}$ & $218 \pm 4.14^{\mathrm{a}}$ & $220 \pm 1.98^{\mathrm{a}}$ \\
\hline & 5 & $46 \pm 1.01^{\mathrm{a}}$ & $64 \pm 1.54^{\mathrm{a}}$ & $100 \pm 4.40^{\mathrm{a}}$ & $134 \pm 5.63^{\mathrm{ab}}$ & $160 \pm 7.04^{\mathrm{bc}}$ & $160 \pm 1.60^{\mathrm{c}}$ & $168 \pm 1.68^{\mathrm{c}}$ & $172 \pm 4.47^{\mathrm{c}}$ \\
\hline & 10 & $43 \pm 1.03^{\mathrm{bc}}$ & $56 \pm 2.41^{\mathrm{b}}$ & $84 \pm 3.53^{\mathrm{b}}$ & $118 \pm 5.78^{\mathrm{b}}$ & $148 \pm 7.25^{\mathrm{c}}$ & $164 \pm 1.97^{\mathrm{c}}$ & $156 \pm 0.31^{\mathrm{c}}$ & $158 \pm 1.58^{\mathrm{d}}$ \\
\hline & 20 & $44 \pm 0.75^{\mathrm{bc}}$ & $61 \pm 1.71^{\mathrm{ab}}$ & $92 \pm 3.40^{\mathrm{ab}}$ & $132 \pm 6.07^{\mathrm{ab}}$ & $176 \pm 6.86^{\mathrm{a}}$ & $218 \pm 1.53^{\mathrm{a}}$ & $222 \pm 9.99^{\mathrm{a}}$ & $222 \pm 8.44^{\mathrm{a}}$ \\
\hline & 30 & $46 \pm 0.92^{\mathrm{a}}$ & $58 \pm 1.74^{\mathrm{b}}$ & $88 \pm 2.64^{\mathrm{b}}$ & $122 \pm 4.76^{\mathrm{b}}$ & $164 \pm 4.59^{\mathrm{ab}}$ & $192 \pm 0.58^{\mathrm{b}}$ & $200 \pm 0.80^{\mathrm{b}}$ & $200 \pm 5.40^{\mathrm{b}}$ \\
\hline \multirow[t]{5}{*}{ TNu64 } & 0 & $47 \pm 0.67^{\mathrm{a}}$ & $60 \pm 1.92^{\mathrm{a}}$ & $92 \pm 0.83^{\mathrm{a}}$ & $128 \pm 5.38^{\mathrm{a}}$ & $168 \pm 5.21^{\mathrm{a}}$ & $190 \pm 7.03^{\mathrm{b}}$ & $218 \pm 4.14^{\mathrm{a}}$ & $220 \pm 1.98^{a}$ \\
\hline & 5 & $44 \pm 1.06^{\mathrm{bc}}$ & $62 \pm 1.98^{\mathrm{a}}$ & $98 \pm 1.08^{\mathrm{ab}}$ & $134 \pm 2.68^{\mathrm{ab}}$ & $174 \pm 8.35^{\mathrm{ab}}$ & $194 \pm 0.58^{b}$ & $186 \pm 8.18^{c}$ & $192 \pm 8.64^{\mathrm{cd}}$ \\
\hline & 10 & $44 \pm 0.44^{\mathrm{bc}}$ & $60 \pm 2.58^{\mathrm{ab}}$ & $94 \pm 3.01^{\mathrm{a}}$ & $136 \pm 0.95^{\mathrm{a}}$ & $168 \pm 5.71^{\mathrm{ab}}$ & $206 \pm 6.18^{\mathrm{a}}$ & $196 \pm 6.08^{\mathrm{bc}}$ & $200 \pm 9.40^{\mathrm{bc}}$ \\
\hline & 20 & $46 \pm 0.69^{\mathrm{ab}}$ & $62 \pm 2.73^{\mathrm{a}}$ & $96 \pm 0.58^{\mathrm{ab}}$ & $134 \pm 5.36^{\mathrm{ab}}$ & $178 \pm 8.54^{\mathrm{a}}$ & $200 \pm 3.60^{\mathrm{ab}}$ & $212 \pm 4.88^{\mathrm{b}}$ & $212 \pm 5.72^{b}$ \\
\hline & 30 & $44 \pm 1.80^{\mathrm{b}}$ & $56 \pm 2.02^{\mathrm{b}}$ & $86 \pm 1.98^{\mathrm{b}}$ & $122 \pm 3.42^{\mathrm{b}}$ & $160 \pm 7.68^{\mathrm{b}}$ & $178 \pm 4.27^{\mathrm{c}}$ & $178 \pm 6.94^{\mathrm{c}}$ & $184 \pm 4.60^{\mathrm{d}}$ \\
\hline \multirow[t]{5}{*}{ TNu66 } & 0 & $47 \pm 0.67^{\mathrm{a}}$ & $60 \pm 1.92^{\mathrm{ab}}$ & $92 \pm 0.83^{\mathrm{a}}$ & $128 \pm 5.38^{\mathrm{a}}$ & $168 \pm 5.21^{\mathrm{ab}}$ & $190 \pm 7.03^{b}$ & $218 \pm 4.141^{\mathrm{a}}$ & $220 \pm 1.98^{b}$ \\
\hline & 5 & $48 \pm 1.01^{\mathrm{a}}$ & $66 \pm 1.98^{\mathrm{a}}$ & $92 \pm 3.04^{\mathrm{a}}$ & $142 \pm 5.54^{\mathrm{a}}$ & $184 \pm 0.37^{\mathrm{a}}$ & $202 \pm 2.22^{\mathrm{b}}$ & $230 \pm 9.43^{\mathrm{a}}$ & $226 \pm 2.49^{b}$ \\
\hline & 10 & $47 \pm 1.32^{\mathrm{a}}$ & $62 \pm 2.42^{\mathrm{ab}}$ & $96 \pm 3.65^{\mathrm{a}}$ & $134 \pm 6.30^{\mathrm{a}}$ & $176 \pm 2.82^{\mathrm{ab}}$ & $190 \pm 6.08^{b}$ & $200 \pm 5.80^{\mathrm{b}}$ & $194 \pm 8.73^{c}$ \\
\hline & 20 & $49 \pm 1.57^{\mathrm{a}}$ & $62 \pm 1.74^{\mathrm{ab}}$ & $100 \pm 1.40^{\mathrm{a}}$ & $138 \pm 3.04^{\mathrm{a}}$ & $176 \pm 5.10^{\mathrm{ab}}$ & $202 \pm 9.70^{\mathrm{b}}$ & $198 \pm 1.78^{b}$ & $200 \pm 0.60^{c}$ \\
\hline & 30 & $49 \pm 1.72^{\mathrm{a}}$ & $60 \pm 1.68^{\mathrm{b}}$ & $90 \pm 4.14^{\mathrm{a}}$ & $130 \pm 3.25^{\mathrm{a}}$ & $172 \pm 0.86^{\mathrm{b}}$ & $216 \pm 9.29^{\mathrm{a}}$ & $226 \pm 1.17^{\mathrm{a}}$ & $250 \pm 6.75^{\mathrm{a}}$ \\
\hline
\end{tabular}

*Mean \pm SD in the same column values not sharing a common superscription letters are significantly different from one another by Duncan's multiple range test $(p<0.05)$.

\begin{tabular}{|c|c|c|c|c|}
\hline Varieties & $\begin{array}{c}\text { Addition } \\
(\%)\end{array}$ & $\begin{array}{c}\text { Weight } \\
(\mathrm{g})^{*}\end{array}$ & $\begin{array}{l}\text { Volume } \\
\text { (c.c.) }\end{array}$ & $\begin{array}{c}\text { Specificvolume } \\
\text { (c.c./g) }\end{array}$ \\
\hline \multirow[t]{5}{*}{ TNu57 } & 0 & $506.67 \pm 7.1^{\mathrm{a}}$ & $2925.00 \pm 63.7^{\mathrm{a}}$ & $5.77 \pm 0.12^{\mathrm{a}}$ \\
\hline & 5 & $508.11 \pm 15.0^{\mathrm{a}}$ & $2908.33 \pm 58.6^{\mathrm{a}}$ & $5.73 \pm 0.19^{\mathrm{a}}$ \\
\hline & 10 & $497.67 \pm 5.5^{\mathrm{a}}$ & $2963.89 \pm 37.7^{\mathrm{a}}$ & $5.96 \pm 0.10^{\mathrm{a}}$ \\
\hline & 20 & $503.33 \pm 7.8^{\mathrm{a}}$ & $2661.11 \pm 110.5^{\mathrm{a}}$ & $5.29 \pm 0.16^{\mathrm{a}}$ \\
\hline & 30 & $501.78 \pm 8.8^{\mathrm{a}}$ & $2669.44 \pm 87.3^{\mathrm{a}}$ & $5.32 \pm 0.23^{\mathrm{a}}$ \\
\hline \multirow[t]{5}{*}{ TNu62 } & 0 & $506.67 \pm 7.1^{\mathrm{a}}$ & $2925.00 \pm 63.7^{\mathrm{a}}$ & $5.77 \pm 0.12^{\mathrm{a}}$ \\
\hline & 5 & $511.67 \pm 5.0^{\mathrm{a}}$ & $2863.89 \pm 199.7^{\mathrm{a}}$ & $5.60 \pm 0.38^{\mathrm{a}}$ \\
\hline & 10 & $502.22 \pm 4.4^{\mathrm{a}}$ & $2966.67 \pm 70.7^{\mathrm{a}}$ & $5.91 \pm 0.17^{\mathrm{a}}$ \\
\hline & 20 & $497.22 \pm 14.6^{\mathrm{a}}$ & $2871.67 \pm 86.3^{\mathrm{a}}$ & $5.78 \pm 0.25^{\mathrm{a}}$ \\
\hline & 30 & $493.89 \pm 6.5^{\mathrm{a}}$ & $2744.44 \pm 185.7^{\mathrm{a}}$ & $5.56 \pm 0.38^{\mathrm{a}}$ \\
\hline \multirow[t]{5}{*}{ TNu64 } & 0 & $506.67 \pm 7.1^{\mathrm{a}}$ & $2925.00 \pm 63.7^{\mathrm{a}}$ & $5.77 \pm 0.12^{\mathrm{a}}$ \\
\hline & 5 & $516.11 \pm 21.9^{\mathrm{a}}$ & $2844.44 \pm 157.5^{\mathrm{a}}$ & $5.53 \pm 0.47^{\mathrm{a}}$ \\
\hline & 10 & $503.89 \pm 5.5^{\mathrm{a}}$ & $2997.22 \pm 8.3^{\mathrm{a}}$ & $5.95 \pm 0.06^{\mathrm{a}}$ \\
\hline & 20 & $510.00 \pm 6.6^{\mathrm{a}}$ & $2750.00 \pm 91.9^{\mathrm{a}}$ & $5.39 \pm 0.16^{\mathrm{a}}$ \\
\hline & 30 & $504.22 \pm 4.6^{\mathrm{a}}$ & $2822.22 \pm 135.5^{\mathrm{a}}$ & $5.60 \pm 0.28^{\mathrm{a}}$ \\
\hline \multirow[t]{5}{*}{ TNu66 } & 0 & $506.67 \pm 7.1^{\mathrm{a}}$ & $2925.00 \pm 63.7^{\mathrm{a}}$ & $5.77 \pm 0.12^{\mathrm{a}}$ \\
\hline & 5 & $500.22 \pm 7.4^{\mathrm{a}}$ & $2950.00 \pm 75.0^{\mathrm{a}}$ & $5.90 \pm 0.09^{\mathrm{a}}$ \\
\hline & 10 & $503.89 \pm 7.7^{\mathrm{a}}$ & $2980.56 \pm 24.3^{\mathrm{a}}$ & $5.92 \pm 0.07^{\mathrm{a}}$ \\
\hline & 20 & $505.89 \pm 8.9^{\mathrm{a}}$ & $2902.78 \pm 101.9^{\mathrm{a}}$ & $5.74 \pm 0.21^{\mathrm{a}}$ \\
\hline & 30 & $506.67 \pm 5.0^{\mathrm{a}}$ & $2766.67 \pm 84.8^{\mathrm{a}}$ & $5.46 \pm 0.15^{\mathrm{a}}$ \\
\hline
\end{tabular}

*Mean \pm SD in the same column values not sharing a common superscription letters are significantly different from one another by Duncan's multiple range test $(\mathbf{p}<0.05)$.

value, which is derived from the area under the curve, is widely used as an index of dough strength [5]. The $\mathrm{W}$ value was significantly reduced by adding sweet potyato paste $(\mathrm{p}<0.05)$.
Extensigraph curves (extensigrams) from different ratio of sweet potato paste addition are shown in Fig. 3. Averaged results of extensibility and resistance to extension after 45 minutes and 90 minutes rest are summarized in Table 2. The addition of sweet potato paste had significant effect on the parameters of extensigraph, but the trend of changes was not obviously. Zaidul et al. [36] reported that the peak viscosity and final viscosity increased with an increase in the mixtures of wheat-sweet potato starches from $10 \%$ to $50 \%$. The RVA parameters of peak viscosity and breakdown were positively correlated with the phosphorus content of potato starch [18]. The peak viscosity and breakdown were negatively correlated with the amylase content [19]. As can be seen in Fig. 4 peak viscosity of amylograph and temperature at peak also were not affected by the addition of sweet potato paste. The findings in this work provide evidence that sweet potato paste could be used as a partial substitute for wheat flour in toast making without significantly changing pasting viscosity.

Effect on dough expansion test of sweet potato paste addition As illustrated in Table 3, the dough volume increase of sweet potato paste addition was similar to that of control group. However, the $10 \%$ addition of sweet potato paste was obviously unable to retain the $\mathrm{CO}_{2}$ which generated by the yeast. This is possibly due to the dough contains less gluten. Dough had a faster volume increase within the first 120 minutes. Then, all dough induced a slow volume increase after 120 minutes fermentation time. Some dough volume of $20 \%$ and $30 \%$ sweet potato paste addition expanse more than the control is due to the 
Table 5. Effect of sweet potato addition on hardness and color* of toast crumb.

\begin{tabular}{|c|c|c|c|c|c|c|c|c|}
\hline \multirow{3}{*}{ Varieties } & \multirow{2}{*}{$\begin{array}{l}\text { Addition amount } \\
\qquad(\mathrm{g} / 100)\end{array}$} & \multicolumn{3}{|c|}{ Hardness } & \multicolumn{4}{|c|}{ Color } \\
\hline & & 1 day & 2 days & 3 days & $\mathrm{L}$ & $\mathrm{a}$ & $\mathrm{b}$ & White index \\
\hline & Control & $101.57 \pm 7.89^{\mathrm{a}}$ & $99.31 \pm 10.19^{\text {cde }}$ & $111.84 \pm 15.37^{\mathrm{g}}$ & $58.7 \pm 0.40^{\mathrm{a}}$ & $-4.4 \pm 0.21^{\mathrm{a}}$ & $10.2 \pm 0.61^{\mathrm{ac}}$ & $57.2 \pm 0.17^{\mathrm{a}}$ \\
\hline TNu 57 & 5 & $61.78 \pm 3.86^{\mathrm{e}}$ & $115.07 \pm 10.45^{\mathrm{bc}}$ & $133.42 \pm 8.28^{\mathrm{def}}$ & $58.2 \pm 1.35^{\mathrm{a}}$ & $-5.0 \pm 0.47^{\mathrm{a}}$ & $11.3 \pm 0.40^{\mathrm{b}}$ & $56.4 \pm 0.17^{\mathrm{a}}$ \\
\hline TNu 57 & 10 & $66.69 \pm 4.71^{\mathrm{de}}$ & $108.51 \pm 6.60^{\text {bcde }}$ & $162.04 \pm 17.74^{\mathrm{abc}}$ & $58.7 \pm 1.17^{\mathrm{a}}$ & $-5.0 \pm 0.55^{\mathrm{a}}$ & $11.4 \pm 0.26^{\mathrm{b}}$ & $56.9 \pm 0.40^{\mathrm{a}}$ \\
\hline TNu 57 & 20 & $60.44 \pm 7.90^{\mathrm{e}}$ & $95.33 \pm 5.74^{\mathrm{e}}$ & $123.30 \pm 8.47^{\mathrm{fg}}$ & $56.6 \pm 3.78^{a}$ & $-5.1 \pm 0.59^{\mathrm{a}}$ & $12.3 \pm 0.75^{\mathrm{b}}$ & $54.6 \pm 0.38^{\mathrm{b}}$ \\
\hline TNu 57 & 30 & $73.58 \pm 8.59^{\text {cde }}$ & $99.20 \pm 7.15^{\text {cde }}$ & $148.04 \pm 7.43^{\text {bde }}$ & $57.7 \pm 3.75^{\mathrm{a}}$ & $-4.6 \pm 0.47^{\mathrm{a}}$ & $13.4 \pm 0.91^{\mathrm{a}}$ & $55.4 \pm 0.11^{\mathrm{b}}$ \\
\hline TNu 62 & 5 & $77.23 \pm .80^{\mathrm{bcd}}$ & $97.28 \pm 9.53^{\mathrm{de}}$ & $130.97 \pm 5.90^{\mathrm{efg}}$ & $58.6 \pm 4.53^{\mathrm{a}}$ & $-4.8 \pm 0.53^{\mathrm{a}}$ & $10.8 \pm 0.87^{\mathrm{bc}}$ & $56.9 \pm 0.34^{\mathrm{b}}$ \\
\hline TNu 62 & 10 & $72.95 \pm 7.30^{\text {cde }}$ & $102.47 \pm 9.57^{\text {bcde }}$ & $134.60 \pm 12.29^{\mathrm{def}}$ & $57.6 \pm 3.35^{\mathrm{a}}$ & $-4.7 \pm 0.47^{\mathrm{a}}$ & $12.1 \pm 1.66^{\mathrm{ab}}$ & $55.7 \pm .39^{\mathrm{ab}}$ \\
\hline TNu 62 & 20 & $76.61 \pm .91^{\mathrm{bcd}}$ & $109.52 \pm 6.95^{\text {bcde }}$ & $144.78 \pm 7.66^{\mathrm{cde}}$ & $55.8 \pm 0.51^{\mathrm{a}}$ & $-4.5 \pm 0.56^{\mathrm{a}}$ & $12.4 \pm 0.55^{\mathrm{ab}}$ & $53.9 \pm 0.43^{\mathrm{b}}$ \\
\hline TNu 62 & 30 & $69.95 \pm 6.30^{\text {cde }}$ & $101.99 \pm 3.52^{\text {bcde }}$ & $145.62 \pm 7.03^{\text {bcde }}$ & $55.6 \pm 4.00^{\mathrm{a}}$ & $-4.6 \pm 0.06^{\mathrm{a}}$ & $12.8 \pm 0.95^{\mathrm{a}}$ & $53.6 \pm 0.27^{b}$ \\
\hline TNu 64 & 5 & $89.17 \pm 1.14^{\mathrm{ab}}$ & $132.04 \pm 10.98^{\mathrm{a}}$ & $166.57 \pm 8.52^{\mathrm{ab}}$ & $57.7 \pm 2.37^{\mathrm{a}}$ & $-4.5 \pm 0.31^{\mathrm{a}}$ & $11.4 \pm 0.76^{\mathrm{c}}$ & $56.0 \pm 0.22^{b}$ \\
\hline TNu 64 & 10 & $97.89 \pm 3.00^{\mathrm{a}}$ & $133.38 \pm 4.94^{\mathrm{a}}$ & $176.72 \pm 7.18^{\mathrm{a}}$ & $56.2 \pm 4.06^{\mathrm{ab}}$ & $-3.9 \pm 0.64^{\mathrm{ab}}$ & $12.2 \pm 0.60^{c}$ & $54.4 \pm 0.11^{\mathrm{c}}$ \\
\hline TNu 64 & 20 & $96.84 \pm 5.42^{\mathrm{a}}$ & $107.01 \pm 4.80^{\text {bcde }}$ & $153.40 \pm 13.17^{\mathrm{bcd}}$ & $53.7 \pm 0.93^{\mathrm{bc}}$ & $-3.3 \pm 0.21^{b c}$ & $13.2 \pm 0.15^{\mathrm{b}}$ & $51.7 \pm 0.26^{\mathrm{c}}$ \\
\hline TNu 64 & 30 & $81.77 \pm 8.69^{\mathrm{bc}}$ & $116.24 \pm 9.18^{\mathrm{b}}$ & $152.86 \pm 10.45^{\mathrm{bcd}}$ & $51.7 \pm 0.60^{\mathrm{c}}$ & $-2.8 \pm 0.35^{\mathrm{c}}$ & $14.1 \pm 0.23^{\mathrm{a}}$ & $49.6 \pm 0.40^{\mathrm{c}}$ \\
\hline TNu66 & 5 & $82.34 \pm 0.15^{\mathrm{bc}}$ & $111.50 \pm 5.28^{\mathrm{bcd}}$ & $149.72 \pm 8.64^{\text {bcde }}$ & $57.4 \pm 0.82^{\mathrm{ab}}$ & $-4.6 \pm 0.31^{\mathrm{a}}$ & $10.9 \pm 0.36^{\mathrm{c}}$ & $55.8 \pm 0.45^{\mathrm{b}}$ \\
\hline TNu66 & 10 & $88.46 \pm 3.78^{\mathrm{ab}}$ & $116.10 \pm 7.24^{\mathrm{b}}$ & $152.68 \pm 8.70^{\mathrm{bcd}}$ & $56.3 \pm 1.47^{\mathrm{ab}}$ & $-4.2 \pm 0.25^{\mathrm{a}}$ & $12.4 \pm 0.61^{\mathrm{b}}$ & $54.4 \pm .27^{\mathrm{ab}}$ \\
\hline TNu66 & 20 & $80.44 \pm .58^{\text {bcd }}$ & $106.57 \pm 7.20^{\text {bcde }}$ & $132.79 \pm 6.39^{\operatorname{defg}}$ & $54.4 \pm 2.91^{\mathrm{bc}}$ & $-3.3 \pm 0.42^{\mathrm{b}}$ & $13.7 \pm 1.05^{\mathrm{a}}$ & $52.3 \pm .16^{\mathrm{bc}}$ \\
\hline TNu66 & 30 & $79.41 \pm .79^{\text {bcd }}$ & $104.95 \pm 6.67^{\text {bcde }}$ & $118.68 \pm 8.69^{\mathrm{fg}}$ & $52.3 \pm 3.25^{\mathrm{c}}$ & $-3.0 \pm 0.50^{\mathrm{b}}$ & $14.3 \pm 0.58^{\mathrm{a}}$ & $50.1 \pm 0.06^{\mathrm{c}}$ \\
\hline
\end{tabular}

*Mean \pm standard deviation in the same column values not sharing a common superscription letters are significantly different from one another by Duncan's multiple range test $(\mathbf{p}<0.05)$.

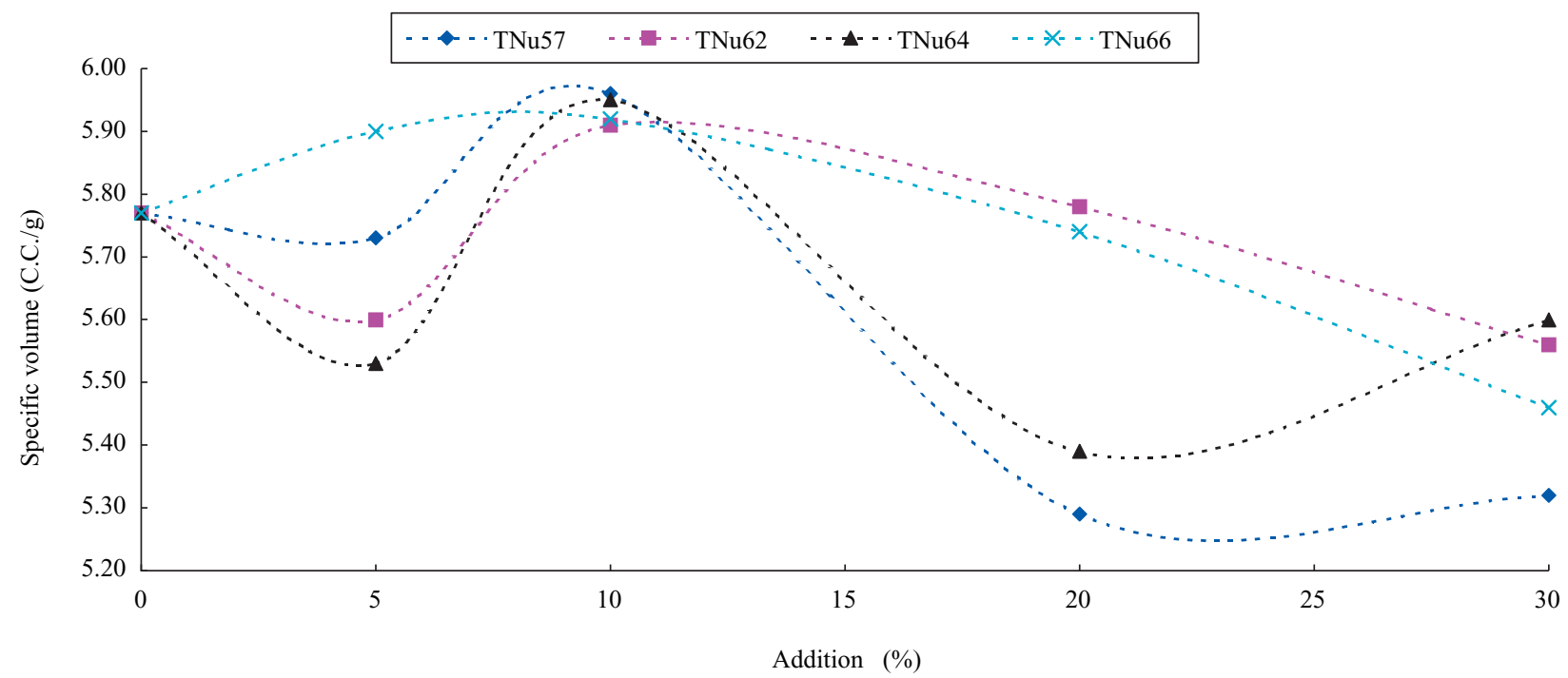

Fig. 5. Effect of various sweet potato paste addition on specific volume of toast.

diluted gluten forming thin gas bubbles, so its final volume is higher than the control group.

Averaged results of toast uniformity calculated by width divided height are not found any significant difference among various groups. Higher loaf weight and volume are desired by bakers on toast at the addition of different flour substitutes. Therefore, toast weight reduction after adding substitutes is an undesirable economic quality to the baking industries as customers often get attracted to toast loaf with higher weight and volume believing that it is more valuable for the same price. Loaf weight is affected by the quantity of dough baked and the amount of moisture and carbon dioxide diffused out of the loaf during baking. Loaf volume is determined by the quantity and quality of protein in the flour [22] and proofing time [37]. The slight decrease in toast specific volume seems relate to diluted gluten concentration of the toast. The addition of $10 \%$ sweet potato paste showed specific volumes slightly higher than other groups ( $>0.05$ ) (Fig. 5). As can be seen, the addition of $10 \%$ sweet potato paste led to the highest toast specific volume, while $30 \%$ addition was the least ( $\mathrm{p}>0.05)$ (Table 4$)$.

Chen [3] reported twenty percentage of sweet potato flour could be added in dough without decreasing volume of bread. Loaf volume significantly decreases with the addition more than $20 \%$ of sweet potato flour. Results of our pre-experiment suggested toast made by adding all sweet potato paste up to $30 \%$ had acceptable qualities. In the appearance of toast, the $40 \%$ addition of sweet potato paste was not good at all among all the tested varieties. All toast could not maintain its shape very well. 
Table 6. Effect of various sweet potato paste addition on sensory evaluation* of toast.

\begin{tabular}{lcccc}
\hline Varieties & Addition\% & Texture** & Color & Acceptance \\
\hline TNu57 & 0 & $2.8 \pm 0.6^{\mathrm{b}}$ & $4.2 \pm 0.8^{\mathrm{a}}$ & $3.6 \pm 0.3^{\mathrm{b}}$ \\
& 5 & $4.4 \pm 0.5^{\mathrm{a}}$ & $4.5 \pm 0.3^{\mathrm{a}}$ & $4.8 \pm 0.4^{\mathrm{a}}$ \\
& 10 & $4.6 \pm 0.4^{\mathrm{a}}$ & $4.1 \pm 0.4^{\mathrm{a}}$ & $5.2 \pm 0.6^{\mathrm{a}}$ \\
& 20 & $4.5 \pm 0.7^{\mathrm{a}}$ & $4.5 \pm 0.5^{\mathrm{a}}$ & $4.6 \pm 0.4^{\mathrm{a}}$ \\
& 30 & $4.6 \pm 0.3^{\mathrm{a}}$ & $4.3 \pm 0.6^{\mathrm{a}}$ & $5.0 \pm 0.3^{\mathrm{a}}$ \\
\hline TNu62 & 0 & $2.8 \pm 0.6^{\mathrm{c}}$ & $4.2 \pm 0.8^{\mathrm{a}}$ & $3.6 \pm 0.3^{\mathrm{b}}$ \\
& 5 & $4.4 \pm 0.5^{\mathrm{b}}$ & $5.1 \pm 0.5^{\mathrm{a}}$ & $4.3 \pm 0.5^{\mathrm{a}}$ \\
& 10 & $4.8 \pm 0.4^{\mathrm{ab}}$ & $4.8 \pm 0.6^{\mathrm{a}}$ & $4.4 \pm 0.4^{\mathrm{a}}$ \\
& 20 & $5.5 \pm 0.7^{\mathrm{a}}$ & $5.3 \pm 0.2^{\mathrm{a}}$ & $5.5 \pm 0.3^{\mathrm{a}}$ \\
\hline TNu64 & 30 & $5.6 \pm 0.4^{\mathrm{a}}$ & $5.0 \pm 0.5^{\mathrm{a}}$ & $5.3 \pm 0.7^{\mathrm{a}}$ \\
& 0 & $2.8 \pm 0.6^{\mathrm{c}}$ & $4.2 \pm 0.8^{\mathrm{a}}$ & $3.6 \pm 0.3^{\mathrm{c}}$ \\
& 5 & $5.2 \pm 0.3^{\mathrm{b}}$ & $4.8 \pm 0.5^{\mathrm{a}}$ & $5.0 \pm 0.4^{\mathrm{b}}$ \\
& 10 & $5.1 \pm 0.5^{\mathrm{b}}$ & $4.3 \pm 0.9^{\mathrm{a}}$ & $5.2 \pm 0.3^{\mathrm{b}}$ \\
& 20 & $6.2 \pm 0.2^{\mathrm{a}}$ & $4.0 \pm 1.1^{\mathrm{a}}$ & $5.8 \pm 0.2^{\mathrm{a}}$ \\
& 30 & $6.5 \pm 0.3^{\mathrm{a}}$ & $5.1 \pm 0.9^{\mathrm{a}}$ & $5.8 \pm 0.3^{\mathrm{a}}$ \\
\hline TNu66 & 0 & $2.8 \pm 0.6^{\mathrm{b}}$ & $4.2 \pm 0.8^{\mathrm{a}}$ & $3.6 \pm 0.3^{\mathrm{b}}$ \\
& 50 & $5.5 \pm 0.4^{\mathrm{a}}$ & $4.0 \pm 1.1^{\mathrm{a}}$ & $5.5 \pm 0.4^{\mathrm{a}}$ \\
& 10 & $5.6 \pm 0.6^{\mathrm{a}}$ & $4.8 \pm 1.5^{\mathrm{a}}$ & $5.7 \pm 0.6^{\mathrm{a}}$ \\
& 20 & $6.4 \pm 0.5^{\mathrm{a}}$ & $5.0 \pm 0.5^{\mathrm{a}}$ & $6.1 \pm 0.5^{\mathrm{a}}$ \\
& $6.5 \pm 0.1^{\mathrm{a}}$ & $5.0 \pm 1.2^{\mathrm{a}}$ & $6.2 \pm 0.3^{\mathrm{a}}$ \\
\hline
\end{tabular}

*The data were averages of triplicate experiments, and in the same column followed by different letters are significantly different $(\mathbf{p}<\mathbf{0 . 0 5})$.

**Texture: 1-very hard, 4-moderate, 7-very soft.

Color: 1-dislike very, 4-moderate, 7-like very much.

Acceptance: 1-very poor, 4-moderate, 7-very good.

Therefore, the upper limit for sweet potato paste adding in this research was $30 \%$. When $30 \%$ sweet potato paste was added, the resulting toast was darker than the control as confirmed by its WI value (Table 5). This was especially detected in $20 \%$ and $30 \%$ addition of TNu64 and TNu66 groups. The values of the tristimulus color parameters L, a, and b of toast crumb as affected by sweet potato paste addition are shown in Table 5 . These values ranged between 51 to $58,-5$ to -2 and 10 to 14 , respectively. The crumb color parameter $\mathrm{L}$ reduced with increasing ratio of sweet potato paste addition (TNu64 and TNu66). This is expected because as the rate of browning pigment formation increase with the color of orange from sweet potato paste. The white index (WI) of the toast crumb ranged from 49 to 57 . The WI was negatively affected by sweet potato paste addition $(\mathrm{p}<0.05)$ while addition of $\mathrm{TNu} 57$ sweet potato paste $(5 \%$ and $10 \%)$ was less significant $(\mathrm{p}>0.05)$ (Table 5). The results also indicate that toast crumb color can be essentially be described as a composite of red and yellow pigments.

Erkan et al. [8] had shown that instrumental measurement of baked products' color is an inevitable quality check that could be used in determing the effects of ingredient or product formulation, process variable and storage conditions on baked products. It is shown crumb color characteristics are liable to differ significantly in higher sweet potato paste adding samples. Hardness is commonly used as an index to determine bread quality, as change in hardness is frequently accompanied with loss of resilience during storage [30]. The effect of sweet potato paste addition on crumb hardness of toast is shown in Table 5. In general, addition of sweet potato paste had significantly softer crumb texture than the control. Only fresh toast made with addition of TNu64 sweet potato didn't show a mark decrease in hardness (Table 5). When addition percentage of sweet potato paste increases, crumb hardness decreases. Meanwhile, results from the hardness of bread were found to increase with increased green tea extract concentration in both sensory evaluation and instrumental analysis [34]. The crumb hardness evolution after one day storage showed bread staling rate of sweet potato paste addition group is faster than the control group. Hardness of toasted made with TNu57 sweet potato paste addition is softer than other varieties at first day baking, but there is no difference among various groups after one day storage. It seems the addition of TNu57 is softer than other group after one day storage.

Toast prepared with TNu57 sweet potato paste addition produced the lowest hardness, which might relate to the high maltose content in the TNu57. As can be seen in Table 5, TNu57 showed a great effect in delaying toast hardening during one day storage. When $20 \%$ of TNu57 sweet potato paste was added, crumb hardness decreased, showing, at the first day, a hardening effect between $5 \%$ and $20 \%$ with respect to those of control. These effects might associate with the differences in water binding capacities related to water loss during storage of the different varieties, and with their interaction with starch, that would affect the starch retrogradation. But results obtained with the hardness of one day storage were not different among control and tested groups. Addition of sweet potato paste in the elaboration of toasts to give products of very soft and with good panelist acceptance for the baking day can be achieved. Shittu et al. [29] found that higher crumb moisture had a positive correlation with crumb softness. However, the relationship was not significant $(\mathrm{p}>0.05)$. It must also be mentioned that baking temperature and time factors influences crumb moisture retention capacity and hardness of bread crumb [7], since the toasts have been baked at same baking temperature and time.

The comparison of storage time effect on hardness of crumb between sweet potato varieties and addition content shows storage time was clearly the most important parameter influencing crumb hardness in all parameters. Stepwise multiple regression was implemented to establish the relationship between two storage times and sweet potato varieties with different addition percentage. In tukey's test the ideal sweet potato variety TNu57 would have the softest toast product at first baking day. The results of these analysis illustrated that the TNu57 had a strong effect as well as a suggestion of softening the crumb hardness of toast which implied that high maltose content in TNu57 sweet potato might decrease the hardness of bread. However, the effect of decrease hardness on crumb at second day storage was not significant. TNu57 still showed its crumb hardness is the lowest one $(p>0.05)$. The significance of hardness of toast crumb in discriminating between the addition percentage of TNu57 and storage was analyzed again using analysis of variance (ANOVA), and tukey's honestly significant difference (HSD) test (significance of differences at $\mathrm{p}<0.05$ ).

In general, the hardness of toast crumb could be modified by using sweet potato paste addition, because the percentage of additions were significantly different $(\mathrm{p}<0.05)$ from the control bread without sweet potato addition at first baking day. Hard- 
ness of toast crumb could be softened with $5 \%$ to $20 \%$ sweet potato (TNu57) paste. None of the softening effect of sweet potato addition bread assessed at the second day of storage was significantly different from each other.

Average results of sensory evaluation attributes of sweet potato paste addition toast are summarized in Table 6. As can be seen, addition of sweet potato paste led to a higher texture and overall acceptance toast, while the addition of $30 \%$ sweet potato was the most flavored. In general, all panelists preferred with sweet potato addition $(\mathrm{p}<0.05)$ on texture and acceptance. In spite of this the hedonic panelists did not find any significant difference in preference with regard to color.

\section{CONCLUSION}

The increase addition of sweet potato paste showed a decrease in Farinograph absorption and mixing time. Toasts manufactured with sweet potato paste addition had in general lower tenacity, extensibility, distortion and deformation energy of alveograph than the control group. Extensibility and resistance to extension of extensigraph were affected by sweet potato paste addition. The addition of various sweet potato paste doesn't influence the peak viscosity of amylograph. Among the four sweet potato varieties, TNu57 was the best variety for toast making. In the dough expansion test, the addition of $10 \mathrm{~g} / 100 \mathrm{~g}$ sweet potato paste went through a maximum expansion. This study showed that broad diversity in the parameters of extensigraph existed in both sweet potato varieties and amount of addition. In the sensory evaluation of toast texture, the $20 \%$ addition of TNu57 was softest among the tested group. The hardness of toast crumb decreased with increasing addition of sweet potato paste. The crumb hardness of three days storage was influenced by the sweet potato variety. In general, toast made by adding TNu57 sweet potato paste had softer texture than other groups. The presence of sweet potato paste seems to decrease the L value and white index of toast crumb, but it also increase the acceptance of the toast appearance.

\section{REFERENCES}

1. AACC, Approved Methods of the American Association of Cereal Chemists, 10th ed., St. Paul, Minnesota (1995).

2. AOAC, Official Methods of Analysis, 16th ed., Arlington, Virginia: Association Official Analytical Chemists (1995).

3. Chen, K. L. and Chiang, W. C., "Manufacture and quality improvement of composite bread made from sweet potato flour and wheat flour," Food Science (Taiwan), Vol. 11, pp. 66-77 (1984).

4. Ciacco, C. F. and D'Appolonia, B. L., "Baking studies with cassava and yam. II Rheological and baking studies of tuber-wheat flour blends," Cereal Chemistry, Vol. 55, No. 4, pp. 423-435 (1978).

5. CIGI, "Breadmaking technology," Chapter D-4. Grains \& Oilseeds, Handling, Marketing, Processing Volume II. Winnipeg, Manitoba: Canada: Canadian International Grains Institute, pp. 655-696 (1993).

6. Dexter, J. E., Preston, K. R., Tweed, A. P., Kilborn, R. H., and Tipples, K. H.. "Relationship of flour starch damage and flour protein to the quality of Brazilian-style hearth bread and remix pan bread produced from hard spring wheat," Cereal Foods World, Vol. 30, pp. 511-514 (1985).

7. Eggleston, G., Omoaka, P. E., and Arowosegbe, A. U., "Flour starch and composite breadmaking quality of various cassava clones," Journal of the Science of Food and Agriculture, Vol. 62, pp. $49-59$ (1993).
8. Erkan, H., Celik, S., Bilgi, B., and Koskel, H., "A new approach for the utilization of barley in food products: barley tarhana," Food Chemistry, Vol. 97, pp. 12-18 (2006).

9. FAO, 1997 Production yearbook, Rome, Italy: Food and Agricultural Organization (1997).

10. Greene, J. L. and Bovell-Benjamin, A. C., "Macroscopic and sensory evaluation of bread supplemented with sweet-potato flour,' Journal Food Science, Vol. 69, pp. SNQ167-173 (2004).

11. Hagenimana, V., Carey, E., Low, J., Gichuki, S., Owori, C., Oyunga, A., and Malinga, J. N., Sweet potato postharvest in East Africa. Lima, Peru: CIP Library. Available from: CIP Subproject Annual Progress Report (1993).

12. Hamed, M. G. E., Refai, F. Y., Hussein, M. F., and El-Samahy, S. K., "Effect of adding sweet potato flour to wheat flour on physical dough properties and baking," Cereal Chemistry, Vol. 50(2), pp. 140-146 (1973).

13. Hsu, C. L., Hurang, S. L., Chen, W., Weng, Y. M., and Tseng, C. Y., "Qualities and antioxidant properties of bread as affected by the incorporation of yam flour in the formulation," International Journal of Food Science \& Technology, Vol. 39, No. 2, pp. 231-238 (2004).

14. Jones, R. W. and Erlander, S. R., "Interactions between wheat proteins and dextrans," Cereal Chemistry, Vol. 44, pp. 447-453 (1967).

15. Kruger, J. E., Morgan, B., Preston, K. R., and Matsuo, R. R., "Evaluation of some characteristics of Chinese steamed buns prepared from Canadian wheat flours," Canadian Journal of Plant Science, Vol. 72, pp. 369-375 (1982).

16. Lazaridou, A., Duta, D., Papageorgiou, M., Belc, N., and Biliaderis, C. G., "Effects of hydrocolloids on dough rheology and bread quality parameters in gluten-free formulations," Journal of Food Engineering, Vol. 79, pp. 1033-1047 (2007).

17. Lee, M. H., "Aroma improvement of the sweet potato flour composite bread and manufacturing of the sweet potato paste composite bread," Journal of the Chinese Agricultural Chemical Society, Vol. 23, No. 1/2, pp. 133-139 (1985).

18. Noda, S., Tsuda, S., Mori, M., Takigawa, S., Endo, C. M., and Saito, K. "The effect of harvested dates on the starch properties cultivars," Food Chemistry, Vol. 86, pp. 119-125 (2004).

19. Noda, T., Fujikami, S., Mura, H., Fukushima, M., Takigawa, S., and Endo, C. M., "Effect of potato starch characteristics on the textureal properties of Korean-style cold noodles made from wheat flour and potatop starch blends," Food Science and Technology Research, Vol. 12, pp. 278-283 (2006).

20. Olatunji, O. and Akinrele, I. A., "Comparative rheological properties and bread qualities of wheat flour diluted with tropical tuber and bread fruit flours," Cereal Chemistry, Vol. 55, No.1, pp. 1-6 (1978).

21. Othira, J., Bhattacharjee, M., and Wanjama, J. K., "Evaluation of dough properties of selected composite wheat flours," Cereal Research Communications, Vol. 32, No. 4, pp. 533-540 (2004).

22. Ragaee, S. and Abdel-Aal, E. S. M., "Pasting properties of starch and protein in selected cereals and quality of their products," Food Chemistry, Vol. 95, pp. 9-18 (2006).

23. Rasper, V., Rasper, J., and Mabey, G. L., "Functional properties of non-wheat flour substitutes in composite flours. I. The effect of non-wheat starches in composite doughs," Canadian Institute of Food Science and Technology Journal, Vol. 7, No. 2, pp. 86-97 (1974).

24. Ravi, V., Aked, J., and Balagopalan, C., "Review on tropical root and tuber crops. I storage methods and quality changes," Critical Review in Food Science and Nutrtion, Vol. 36, pp. 661-709 (1996).

25. Rehm, S. and Espig, G., The Cultivated Plants of the Tropics and Subtropics, Weikersheim (Germany): Markgraf (1991).

26. Sangnark, A. and Noomhrm, A., "Effect of dietary fiber from sugarcane bagasse and sucrose ester on dough and bread properties," LebensmittelWissenschaft und-Technologie, Vol. 37, pp. 697-704 (2004).

27. Sangnark, A. and Noomhorm, A., "Effect of particle sizes on functional properties of dietary fibre prepared from sugarcane bagasse," Food Chemistry, Vol. 80, pp. 221-229 (2003).

28. SAS, PC-SAS User's Guide, North Carolina: Statistical Analysis System Institute, Inc (1995).

29. Shittu, T. A., Raji, A. O., and Sanni, L. O., "Bread from composite cassava-wheat flour: I. Effect of baking time and temperature on some physical properties of bread loaf," Food Research International, Vol. 40, pp. 280-290 (2007).

30. Spices, R., "Application of rheology in the bread industry," Dough 
Rheology and Baked Product Texture, New York, Van Nostrand Reinhold, pp. 343-361 (1990).

31. Tipples, K. H. Meredith, J. O., and Holas, J., "Factors affecting farinograph and baking absorption II. Relative influence of flour components," Cereal Chemistry, Vol. 55, pp. 652-660 (1978).

32. Wang, F. C. and Sun, X. S., "Frequency dependence of viscoelastic properties of bread crumb and relation to bread staling," Cereal Chemistry, Vol. 79, pp. 108-114 (2002).

33. Wang, J., Rosell, C. M., and Barber, C. B., "Effect of the addition of different fibres on wheat dough performance and bread quality," Food Chemistry, Vol. 79, pp. 221-226 (2002).

34. Wang, R., Zhou, W., and Isabelle, M., "Comparison study of the effect of green tea extract (GTE) on the quality of bread by instrumental analysis and sensory evaluation," Food Research International, Vol. 40, pp. 470-479 (2007).

35. Woolfe, J. A., Sweet Potato: an Untapped Food Resource, Cambridge, UK: Cambridge University Press (1992).

36. Zaidul, I. S. M., Nik Norulaini, N.A., Mohd Omar, A.K., Yamauch, H., and Noda, T., "RVA analysis of mixtures of wheat flour and potato, sweet potato, yam, and cassava starches," Carbohydrat Polymers, Vol. 69, No. 4, pp. 784-791 (2007).

37. Zghal, M. C., Scanlon, M. G., and Sapirstein, H. D., "Cellular structure of bread crumb and its influence on mechanical properties," Journal of Cereal Chemistry, Vol. 36, pp. 167-176. (2002). 